\title{
What's Eating You? Oriental Rat Flea (Xenopsylla cheopis)
}

\author{
Leah Ellis Wells, MD; Dirk M. Elston, MD
}

\section{PRACTICE POINTS}

- Xenopsylla cheopis, the oriental rat flea, is most known for carrying Yersinia pestis, the causative agent of the plague; however, it also is a vector for other bacteria, such as Rickettsia typhi, the species responsible for most cases of murine typhus.

- Despite the perception that it largely is a historical illness, modern outbreaks of plague occur in many parts of the world each year. Because fleas thrive in warm humid weather, global warming threatens the spread of the oriental rat flea and its diseases into previously unaffected parts of the world.

- There has been an effort to control oriental rat flea populations, which unfortunately has been complicated by pesticide resistance in many flea populations. It is important to continue to research the oriental rat flea and the bacterial species it carries in the hopes of finding better methods of controlling the pests and therefore decreasing illness in humans.

- Health care providers should be vigilant in identifying symptoms of flea-borne illnesses. If a patient is displaying symptoms, prompt recognition and antibiotic therapy is critical, particularly for the plague.

The oriental rat flea (Xenopsylla cheopis) is an ectoparasite of small mammals and a vector of many diseases for which humans are incidental hosts. This species of flea is most widely known for carrying Yersinia pestis and Rickettsia typhi, the causative agents of the plague and murine typhus, respectively. Public health issues related to $X$ cheopis may increase in the future as global warming expands the geographic area in which the fleas can survive. A bioterrorist attack of plague also remains a threat. Extensive research is ongoing regarding $X$ cheopis and its interaction with the bacteria it transmits to find better ways of reducing related morbidity and mortality. Traditional control measures include extermination of small mammal hosts, insecticide use to eliminate the flea itself, and use of antibiotics to control the associated diseases. The future may include targeted insecticide usage to prevent the continued development of resistance as well as new methods of reducing transmission of flea-borne diseases that could eliminate the need for chemical insecticides all together.

Cutis. 2020;106:124-126.

$\Lambda$ dult Siphonaptera (fleas) are highly adapted to life on the surface of their hosts. Their small 2- to $10-\mathrm{mm}$ bodies are laterally flattened and wingless. They utilize particularly strong hind legs for jumping up to 150 times their body length and backward-directed spines on their legs and bodies for moving forward through fur, hair, and feathers. Xenopsylla cheopis, the oriental rat flea, lacks pronotal and genal combs and has a mesopleuron divided by internal scleritinization (Figure). These features differentiate the species from its close relatives, Ctenocephalides (cat and dog fleas), which have both sets of combs, as well as Pulex irritans (human flea), which do not have a divided mesopleuron. ${ }^{1,2}$

Flea-borne infections are extremely important to public health and are present throughout the world. Further, humidity and warmth are essential for the life cycle of many species of fleas. Predicted global warming likely will increase their distribution, allowing the spread of diseases they carry into previously untouched areas. ${ }^{1}$ Therefore, it

Dr. Wells is from the Department of Internal Medicine, University of Virginia, Charlottesville. Dr. Elston is from the Department of Dermatology and Dermatologic Surgery, Medical University of South Carolina, Charleston.

The authors report no conflict of interest.

The image is in the public domain.

Correspondence: Leah Ellis Wells, MD, University Medical Associates, UVA Jefferson Park Ave, Medical Office Building, 3rd Floor, 1222 Jefferson Park Ave, Charlottesville, VA 22903 (leah.ellis.wells@gmail.com).

doi:10.12788/cutis.0072 


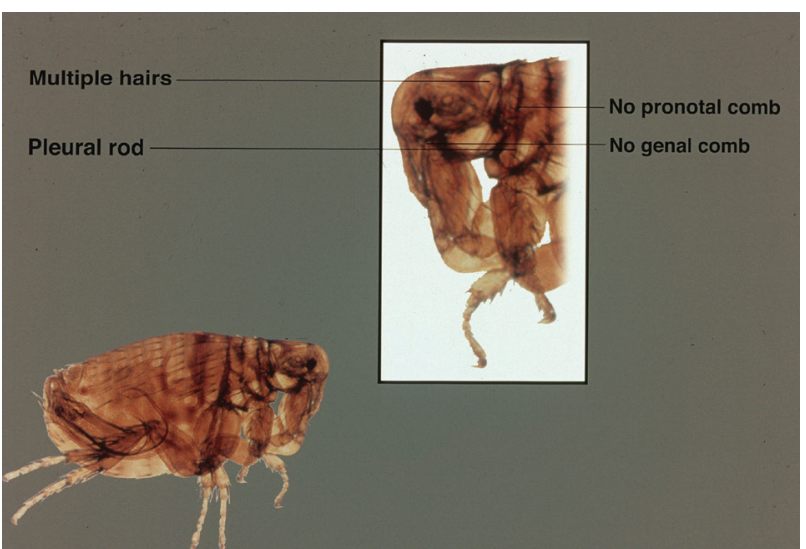

Xenopsylla cheopis.

is important to continue to examine species that carry particularly dangerous pathogens, such as $X$ cheopis.

\section{Disease Vector}

Xenopsylla cheopis primarily is known for being a vector in the transmission of Yersinia pestis, the etiologic agent of the plague. Plague occurs in 3 forms: bubonic, pneumonic, and septicemic. It has caused major epidemics throughout history, the most widely known being the Black Death, which lasted for 130 years, beginning in the 1330s in China and spreading into Europe where it wiped out one-third of the population. However, bubonic plague is thought to have caused documented outbreaks as early as $320 \mathrm{BCE}$, and it still remains endemic today. ${ }^{3,4}$

Between January 2010 and December 2015, 3248 cases of plague in humans were reported, resulting in 584 deaths worldwide. ${ }^{5}$ It is thought that the plague originated in Central Asia, and this area still is a focus of disease. However, the at-risk population is reduced to breeders and hunters of gerbils and marmots, the main reservoirs in the area. In Africa, 4 countries still regularly report cases, with Madagascar being the most severely affected country in the world. ${ }^{5}$ The Democratic Republic of the Congo, Uganda, and Tanzania also are affected. The Americas also experience the plague. There are sporadic cases of bubonic plague in the northwest corner of Peru, mostly in rural areas. In the western United States, plague circulates among wild rodents, resulting in several reported cases each year, with the most recent confirmed case noted in California in August 2020.5,6 Further adding to its relevance, $Y$ pestis is one of several organisms most likely to be used as a biologic weapon. ${ }^{3,4}$

Due to the historical and continued significance of $Y$ pestis, many studies have been performed over the decades regarding its association with $X$ cheopis. It has been discovered that fleas transmit the bacteria to the host in 2 ways. The most well-defined form of transmission occurs after an incubation period of $Y$ pestis in the flea for 7 to 31 days. During this time, the bacteria form a dense biofilm on a valve in the flea foregut - the proventriculus-interfering with its function, which allows regurgitation of the blood and the bacteria it contains into the bite site and consequently disease transmission. The proventriculus can become completely blocked in some fleas, preventing any blood from reaching the midgut and causing starvation. In these scenarios, the flea will make continuous attempts to feed, increasing transmission. ${ }^{7}$ The hemin storage gene, $h m s$, encoding the second messenger molecule cyclic di-GMP plays a critical role in biofilm formation and proventricular blockage. ${ }^{8}$ The phosphoheptose isomerase gene, GmhA, also has been elucidated as crucial in late transmission due to its role in biofilm formation. ${ }^{9}$ Early-phase transmission, or biofilm-independent transmission, has been documented to occur as early as 3 hours after infection of the flea but can occur for up to 4 days..$^{10}$ Historically, the importance of early-phase transmission has been overlooked. Research has shown that it likely is crucial to the epizootic transmission of the plague..$^{10}$ As a result, the search has begun for genes that contribute to the maintenance of $Y$ pestis in the flea vector during the first 4 days of colonization. It is thought that a key evolutionary development was the selective loss-of-function mutation in a gene essential for the activity of urease, an enzyme that causes acute oral toxicity and mortality in fleas. ${ }^{11,12}$ The Yersinia murine toxin gene, Ymt, also allows for early survival of $Y$ pestis in the flea midgut by producing a phospholipase $\mathrm{D}$ that protects the bacteria from toxic by-products produced during digestion of blood. ${ }^{11,13}$ In addition, gene products that function in lipid A modification are crucial for the ability of $Y$ pestis to resist the action of cationic antimicrobial peptides it produces, such as cecropin A and polymyxin B. ${ }^{13}$

Murine typhus, an acute febrile illness caused by Rickettsia typhi, is another disease that can be spread by oriental rat fleas. It has a worldwide distribution. In the United States, $R$ typhi-induced rickettsia mainly is concentrated in suburban areas of Texas and California where it is thought to be mainly spread by Ctenocephalides, but it also is found in Hawaii where spread by $X$ cheopis has been documented. ${ }^{14,15}$ The most common symptoms of rickettsia include fever, headache, arthralgia, and a characteristic rash that is pruritic and maculopapular, starting on the trunk and spreading peripherally but sparing the palms and soles. This rash occurs about a week after the onset of fever. ${ }^{14}$ Rickettsia felis also has been isolated in the oriental rat flea. However, only a handful of cases of human disease caused by this bacterium have been reported throughout the world, with clinical similarity to murine typhus likely leading to underestimation of disease prevalence. ${ }^{15}$ Bartonella and other species of bacteria also have been documented to be spread by $X$ cheopis. ${ }^{16}$ Unfortunately, the interactions of $X$ cheopis with these other bacteria are not as well studied as its relationship with $Y$ pestis. 


\section{Adverse Reactions}

A flea bite itself can cause discomfort. It begins as a punctate hemorrhagic area that develops a surrounding wheal within 30 minutes. Over the course of 1 to 2 days, a delayed reaction occurs and there is a transition to an extremely pruritic, papular lesion. Bites often occur in clusters and can persist for weeks. ${ }^{1}$

\section{Prevention and Treatment}

Control of host animals via extermination and proper sanitation can secondarily reduce the population of $X$ cheopis. Direct pesticide control of the flea population also has been suggested to reduce flea-borne disease. However, insecticides cause a selective pressure on the flea population, leading to populations that are resistant to them. For example, the flea population in Madagascar developed resistance to DDT (dichlorodiphenyltrichloroethane), dieldrin, deltamethrin, and cyfluthrin after their widespread use. ${ }^{17}$ Further, a recent study revealed resistance of $X$ cheopis populations to alphacypermethrin, lambda-cyhalothrin, and etofenprox, none of which were used in mass vector control, indicating that some crossresistance mechanism between these and the extensively used insecticides may exist. With the development of widespread resistance to most pesticides, flea control in endemic areas is difficult. Insecticide targeting to fleas on rodents (eg, rodent bait containing insecticide) can allow for more targeted insecticide treatment, limiting the development of resistance. ${ }^{17}$ Recent development of a maceration protocol used to detect zoonotic pathogens in fleas in the field also will allow management with pesticides to be targeted geographically and temporally where infected vectors are located. ${ }^{18}$ Research of the interaction between vector, pathogen, and insect microbiome also should continue, as it may allow for development of biopesticides, limiting the use of chemical pesticides all together. The strategy is based on the introduction of microorganisms that can reduce vector lifespan or their ability to transmit pathogens. ${ }^{17}$

When flea-transmitted diseases do occur, treatment with antibiotics is advised. Early treatment of the plague with effective antibiotics such as streptomycin, gentamicin, tetracycline, or chloramphenicol for a minimum of 10 days is critical for survival. Additionally, patients with bubonic plague should be isolated for at least 2 days after administration of antibiotics, while patients with the pneumonic form should be isolated for 4 days into therapy to prevent the spread of disease. Prophylactic therapy for individuals who come into contact with infected individuals also is advised. ${ }^{4}$ Patients with murine typhus typically respond to doxycycline, tetracycline, or fluoroquinolones. The few cases of $R$ felis-induced disease have responded to doxycycline. Of note, short courses of treatment of doxycycline are appropriate and safe in young children. The short (3-7 day) nature of the course limits the chances of teeth staining. ${ }^{14}$

\section{REFERENCES}

1. Bitam I, Dittmar K, Parola P, et al. Flea and flea-borne diseases. Int J Infect Dis. 2010;14:E667-E676.

2. Mathison BA, Pritt BS. Laboratory identification of arthropod ectoparasites. Clin Microbiol Rev. 2014;27:48-67.

3. Ligon BL. Plague: a review of its history and potential as a biological weapon. Semin Pediatr Infect Dis. 2006;17:161-170.

4. Josko D. Yersinia pestis: still a plague in the 21st century. Clin Lab Sci. 2004;17:25-29.

5. Plague around the world, 2010-2015. Wkly Epidemiol Rec. 2016; 91:89-93

6. Sullivan K. California confirms first human case of the plague in 5 years: what to know. NBC News website. https://www.nbcnews.com /health/health-news/california-confirms-first-human-case-bubonic -plague-5-years-what-n1237306. Published August 19, 2020. Accessed August 24, 2020.

7. Hinnebusch BJ, Bland DM, Bosio CF, et al. Comparative ability of Oropsylla and Xenopsylla cheopis fleas to transmit Yersinia pestis by two different mechanisms. PLOS Negl Trop Dis. 2017;11:e0005276.

8. Bobrov AG, Kirillina O, Vadyvaloo V, et al. The Yersinia pestis HmsCDE regulatory system is essential for blockage of the oriental rat flea (Xenopsylla cheopis), a classic plague vector. Environ Microbiol. 2015;17:947-959.

9. Darby C, Ananth SL, Tan L, et al. Identification of gmhA, a Yersina pestis gene required for flea blockage, by using a Caenorhabditis elegans biofilm system. Infect Immun. 2005;73:7236-7242.

10. Eisen RJ, Dennis DT, Gage KL. The role of early-phase transmission in the spread of Yersinia pestis. J Med Entomol. 2015;52:1183-1192.

11. Carniel E. Subtle genetic modifications transformed an enteropathogen into a flea-borne pathogen. Proc Natl Acad Sci U S A. 2014;111:1840918410 .

12. Chouikha I, Hinnebusch BJ. Silencing urease: a key evolutionary step that facilitated the adaptation of Yersinia pestis to the flea-borne transmission route. Proc Natl Acad Sci U S A. 2014;111:18709-19714.

13. Aoyagi KL, Brooks BD, Bearden SW, et al. LPS modification promotes maintenance of Yersinia pestis in fleas. Microbiology. 2015;161:628-638.

14. Civen R, NgoV. Murine typhus: an unrecognized suburban vectorborne disease. Clin Infect Dis. 2008;46:913-918

15. Eremeeva ME, Warashina WR, Sturgeon MM, et al. Rickettsia typhi and R. felis in rat fleas (Xenopsylla cheopis), Oahu, Hawaii. Emerg Infect Dis. 2018;14:1613-1615

16. Billeter SA, GundiVAKB, Rood MP, et al. Molecular detection and identification of Bartonella species in Xenopsylla cheopis fleas (Siphonaptera: Pulicidae) collected from Rattus norvecus rats in Los Angeles, California. Appl Environ Microbiol. 2011;77:7850-7852.

17. Miarinjara A, Boyer S. Current perspectives on plague vector control in Madagascar: susceptibility status of Xenopsylla cheopis to 12 insecticides. PLOS Negl Trop Dis. 2016;10:e0004414.

18. Harrison GF, Scheirer JL, Melanson VR. Development and validation of an arthropod maceration protocol for zoonotic pathogen detection in mosquitoes and fleas. J Vector Ecol. 2014;40:83-89. 